\title{
Development of a Taiwan cancer-related fatigue cognition questionnaire: reliability and validity
}

\author{
Shih-Chiung Lai ${ }^{1, *}$,Wei-Chun Lin ${ }^{2, *}$, Chien-Hsin Chen ${ }^{3}$, Szu-Yuan Wu ${ }^{4,5,6,7}$ \\ ${ }^{1}$ Department of Exercise and Health Science, National Taipei University of Nursing and Health Sciences, Taipei, Taiwan \\ ${ }^{2}$ Department of Cancer Research Center, Wan Fang Hospital, Taipei Medical University, Taiwan \\ ${ }^{3}$ Department of Colorectal Surgery, Wan Fang Hospital, Taipei Medical University, Taipei, Taiwan \\ ${ }^{4}$ Institute of Toxicology, College of Medicine, National Taiwan University, Taipei, Taiwan \\ ${ }^{5}$ Department of Radiation Oncology, Wan Fang Hospital, Taipei Medical University, Taipei, Taiwan \\ ${ }^{6}$ Department of Internal Medicine, School of Medicine, College of Medicine, Taipei Medical University, Taipei, Taiwan \\ ${ }^{7}$ Department of Biotechnology, Hungkuang University, Taichung, Taiwan \\ *These authors contributed equally to this work and are joint first authors
}

Correspondence to: Szu-Yuan Wu, email: szuyuanwu5399@gmail.com

Keywords: cancer-related fatigue questionnaire, reliability, validity

Received: October 17, $2016 \quad$ Accepted: February 06, $2017 \quad$ Published: March 16, 2017

Copyright: Lai et al. This is an open-access article distributed under the terms of the Creative Commons Attribution License (CC-BY), which permits unrestricted use, distribution, and reproduction in any medium, provided the original author and source are credited.

\section{ABSTRACT}

Purpose: We prospectively designed a Taiwan cancer-related fatigue cognition questionnaire, version 1.0 (TCRFCQ-V1.0), for Taiwanese patients with cancer and investigated the reliability and validity of this questionnaire.

Results: The completion rate of the TCRFCQ-V1.0 was high ( $97 \%$ of the patients completed all items), and the rate of missing data was low (0.2\%-1.1\% for each item). Moreover, the Cronbach alpha value was $\mathbf{0 . 8 8 9}$. We eliminated 5 items because their respective Cronbach alpha values were higher than the total mean value of Cronbach's alpha. Overall, the TCRFCQ-V1.0 had adequate Cronbach alpha coefficients (range, from 0.882 to 0.889 ). In addition, the results of Bartlett's test were significant (chi-squared, 2390.11; $\boldsymbol{p}<\mathbf{0 . 0 0 1}$ ), indicating the appropriateness of factor analysis. Sampling adequacy was confirmed by the Kaiser-Meyer-Olkin statistic of 0.868. Through exploratory factor analysis, we identified 6 factors with eigenvalues of $>1$, and the scree plot indicated no flattening factors. Overall, 28 items achieved a factor loading of $\geq$ 0.55 .

Materials and Methods: We enrolled patients with cancer who were aged > 18 years, had received a pathological diagnosis of cancer, and had undergone cancer treatments such as surgery, chemotherapy, radiotherapy, or concurrent chemoradiotherapy at a single institute in Taiwan. Of the identified 167 eligible patients, 161 (96.4\%) were approached. Of these patients, $6(7.2 \%)$ declined to participate and $155(92.8 \%)$ were interviewed. The initial 43 items in the TCRFCQ-V1.0 were assessed for ceiling and floor effects.

Conclusions: The TCRFCQ-V1.0 is a reliable and valid instrument for measuring CRF cognition in Taiwanese patients with cancer.

\section{INTRODUCTION}

Cancer-related fatigue (CRF) is a common, distressing, and potentially treatable condition [1]. Although CRF most commonly occurs during active cancer therapy, it may affect patients long after the completion of cancer treatment $[2,3]$. Cancer patients with
CRF could feel extremely tired in community activities, social events, relationshipd, and daily activities [4-6]. Friendship and family time might be loss because patients would spend more time sleeping which may cause school or work miss. Patients with CRF also could lead to mood changes and mental fatigue in some status [7]. CRF can cause cancer patients absent minded, think confused, 
and forget things easily. Financial problems might be encountered by CRF patients who leave from the jobs or cannot keep working full time. Taken together, CRF could reduce patients' quality of life (QOL) and cause them to receive suboptimal cancer treatment [8].

Specific diagnostic criteria have been proposed for defining CRF as an independent entity in the 10th revision International Classification of Diseases (ICD-10) [9]. At least 6 of the 11 criteria must be met for establishing a diagnosis of CRF. In studies including diverse patient populations, approximately $10 \%-26 \%$ of patients were diagnosed as having CRF by using this definition [10]. However, the formal diagnostic criteria outlined in ICD-10 are not widely used, and patients are not necessarily required to meet a minimum number of criteria to receive a clinical diagnosis of CRF. Moreover, guidelines from expert groups, including the National Comprehensive Cancer Network (NCCN), concerning the screening and assessment of CRF do not recommend using the diagnostic criteria outlined in ICD-10 [1, 11, 12].

In Taiwanese cancer patients after cancer therapy, CRF is one of the most popular and suffering symptoms reported in Taiwan. A trusty and effective instrument for measuring CRF and fatigue-related cognition is essential for successful clinical care of Taiwanese patients with cancer who experience CRF. In this study, we prospectively designed a Taiwan CRF cognition questionnaire, version 1.0 (TCRFCQ-V1.0), for Taiwanese patients with cancer and investigated the reliability and validity of this questionnaire.

\section{RESULTS}

\section{Patient characteristics}

Of the identified 167 eligible patients, 161 (96.4\%) were approached. Of these, 6 declined to participate. Thus, we interviewed $155(92.8 \%)$ patients. We did not approach some eligible patients because they were discharged early, had poor communication skills and cognitive impairment, or were missed planned outpatient clinical visit. Three participants were later considered ineligible because they didn't receive cancer therapy, causing a study sample of 148 patients finally. Most of the patients were aged 40-59 years $(n=84,57 \%)$, were men $(n=95,64 \%)$, and had high school education $(n=62,42 \%)$ or higher $(n=66$, $45 \%$ ). We collected most eligible cancer patients in our outpatient cancer clinics $(n=87,59 \%)$. Furthermore, $31 \%$, $19 \%$, and $89 \%$ of the patients received a newly diagnosis of cancer within 3 months, had diagnosis of distant metastases, and were recently under adjuvant therapy, respectively. Breast adenocarcinoma (34\%), lung cancers $(33 \%)$, blood cancers $(7 \%)$, and cancers originated from the digestive system $(26 \%)$ were the greater part of cases.

The rate of the TCRFCQ-V1.0 was completed extremely high $(97 \%$ of the patients completed all questions), and the missing rate was low $(0.2 \%-1.1 \%$ for each question).

\section{Internal reliability}

A satisfactory Cronbach alpha value should range from 0.7 to 0.9 . Our Cronbach alpha value was 0.889 (Table 1). Five items were eliminated because their respective Cronbach alpha values were higher than the total mean value of Cronbach's alpha. Overall, the TCRFCQ-V1.0 had adequate Cronbach alpha coefficients (range, from 0.882 to 0.889 ; Table 1 ).

\section{Construct validity}

The Bartlett's test in the current study were significant (chi-squared, 2390.11; $p<0.001$ ) which indicated the factor analysis were appropriate. The KMO statistic of 0.868 showed the adequate sampling in the study. By using EFA, we identified 6 factors with eigenvalues of $>1$. The scree plot indicated no flattening factors. We identified a six factor model as the most clinically significant for CRF cofnition. Thus, we repeat EFA, and used varimax rotation as a solution of 6 factor. Overall, a factor loading of $\geq 0.55$ was achieved in 28 items. In addition, based on the high currency of not fulfilled and the clinical significance between cancer patients, 7 additional items were maintained. The factor analysis were not contributed by these 7 additional factors (Table 2). Furthermore, there were no $\geq 0.55$ factor loading and $\geq 15 \%$ levels of cognition in the 7 additional factors. Consequently, we removed the 7 items considered not as clinical significance from the tool. The detailed factor loadings of the items are listed in Table 2. The TCRFCQ-V1.0 includes 6 dimensions of CRF, namely unfocused life, attribution cognition, help expectation, treatment helplessness, physician-patient communication, and life power. These 6 dimensions accounted for $67 \%$ of the total variance (Table 3). Four EFA procedures are summarized in Table 4. The findings indicate that our newly developed TCRFCQ-V1.0 is a trusty and appropriate instrument for measuring CRF cognition in Taiwanese cancer patients.

\section{DISCUSSION}

All patients with cancer should be screened for CRF in the first visit; at the finish of primary treatment, as indicated during clinical follow-up survivor care when the diagnosis of advanced disease is made; and at each therapy visit [1]. Screening should be performed and documented using a quantitative or semiquantitative assessment. One example is the visual analog scale [1, 11-13]. Other instruments have been developed and validated for quantifying CRF [14]. Some of the more commonly used and satisfactorily validated instruments are the fatigue and 
Table 1: Scale of the Taiwan cancer-related fatigue questionnaire verified through item and reliability analyses

\begin{tabular}{|c|c|c|c|c|}
\hline \multirow{2}{*}{ Item Number } & \multirow{2}{*}{$\begin{array}{l}\text { Critical ratio test } \\
\text { (CR; i.e., } t \text { value) }\end{array}$} & \multicolumn{2}{|c|}{$\begin{array}{c}\text { Homogeneity test } \\
\text { ( } \alpha \text { value of the total reliability analysis is } 0.889 \text { ) }\end{array}$} & \multirow{2}{*}{ Remark } \\
\hline & & $\begin{array}{l}\text { Correlation between items } \\
\text { and total scale }(\mathbf{R})\end{array}$ & Cronbach's $\alpha$ value & \\
\hline Q1 & 3.763 & 0.320 & 0.888 & \\
\hline Q2 & 3.117 & 0.364 & 0.887 & \\
\hline Q3 & 4.802 & 0.428 & 0.886 & \\
\hline Q4 & 3.329 & 0.310 & 0.888 & \\
\hline Q5 & 3.373 & 0.329 & 0.888 & \\
\hline Q6 & 4.642 & 0.362 & 0.887 & \\
\hline Q7 & 3.540 & 0.337 & 0.887 & \\
\hline Q8 & 6.248 & 0.516 & 0.885 & \\
\hline Q9 & 6.108 & 0.500 & 0.886 & \\
\hline $\mathrm{Q} 10$ & $-0.220(\mathrm{t}<3)$ & 0.008 & $0.891(>0.889)$ & delete \\
\hline Q11 & 8.678 & 0.581 & 0.884 & \\
\hline Q12 & 9.362 & 0.675 & 0.883 & \\
\hline Q13 & 5.598 & 0.421 & 0.887 & \\
\hline Q14 & 2.413 & 0.216 & 0.889 & \\
\hline Q15 & 6.642 & 0.543 & 0.885 & \\
\hline Q16 & 7.674 & 0.548 & 0.885 & \\
\hline Q17 & 2.778 & 0.280 & 0.888 & \\
\hline Q18 & 2.778 & 0.223 & 0.889 & \\
\hline Q19 & 7.810 & 0.557 & 0.885 & \\
\hline Q20 & 11.960 & 0.681 & 0.883 & \\
\hline Q21 & $2.619(\mathrm{t}<3)$ & 0.447 & $0.906(>0.889)$ & delete \\
\hline $\mathrm{Q} 22$ & 10.778 & 0.683 & 0.882 & \\
\hline Q23 & 10.215 & 0.711 & 0.883 & \\
\hline Q24 & 9.584 & 0.617 & 0.884 & \\
\hline Q25 & 7.119 & 0.507 & 0.886 & \\
\hline Q26 & 8.725 & 0.618 & 0.884 & \\
\hline Q27 & 13.835 & 0.744 & 0.882 & \\
\hline Q27 & 13.835 & 0.744 & 0.882 & \\
\hline Q28 & 11.462 & 0.715 & 0.882 & \\
\hline Q29 & 9.783 & 0.704 & 0.882 & \\
\hline Q30 & 7.537 & 0.643 & 0.883 & \\
\hline Q31 & 9.885 & 0.658 & 0.884 & \\
\hline Q32 & $-8.376(\mathrm{t}<3)$ & -0.586 & $0.899(>0.889)$ & delete \\
\hline Q33 & 8.492 & 0.609 & 0.884 & \\
\hline Q34 & $-8.019(\mathrm{t}<3)$ & -0.552 & $0.898(>0.889)$ & delete \\
\hline Q35 & $-0.266(\mathrm{t}<3)$ & -0.037 & $0.892(>0.889)$ & delete \\
\hline Q36 & 7.161 & 0.517 & 0.885 & \\
\hline Q37 & 10.269 & 0.591 & 0.884 & \\
\hline Q38 & 8.879 & 0.594 & 0.884 & \\
\hline Q39 & 5.439 & 0.493 & 0.886 & \\
\hline $\mathrm{Q} 40$ & 7.218 & 0.513 & 0.886 & \\
\hline Q41 & 5.459 & 0.448 & 0.886 & \\
\hline Q42 & 5.397 & 0.460 & 0.886 & \\
\hline Q43 & 4.907 & 0.433 & 0.887 & \\
\hline
\end{tabular}

Note: The highest and lowest values were tested in the critical ratio test. 
Table 2: Exploratory factor analysis of items of the Taiwan cancer-related fatigue questionnaire

CRF summary with the maximum variation method

\begin{tabular}{|c|c|c|c|c|c|c|c|}
\hline $\begin{array}{c}\text { New items } \\
\text { (re-edited ordering) }\end{array}$ & $\begin{array}{l}\text { Unfocused } \\
\text { life }\end{array}$ & $\begin{array}{l}\text { Attribution } \\
\text { cognition }\end{array}$ & $\begin{array}{c}\text { Help } \\
\text { expectation }\end{array}$ & $\begin{array}{c}\text { Treatment } \\
\text { helplessness }\end{array}$ & $\begin{array}{l}\text { Physician-patient } \\
\text { communication }\end{array}$ & Life power & Commonality \\
\hline Q1 & 0.834 & 0.046 & 0.113 & 0.222 & 0.136 & 0.118 & 0.792 \\
\hline Q2 & 0.813 & 0.174 & 0.119 & 0.178 & 0.184 & 0.044 & 0.773 \\
\hline Q3 & 0.801 & 0.102 & 0.139 & 0.127 & 0.169 & 0.263 & 0.785 \\
\hline Q4 & 0.760 & 0.032 & 0.175 & 0.162 & 0.134 & 0.070 & 0.659 \\
\hline Q5 & 0.649 & -0.008 & 0.086 & 0.290 & 0.118 & 0.326 & 0.633 \\
\hline Q6 & 0.613 & -0.008 & 0.215 & 0.366 & 0.243 & 0.182 & 0.649 \\
\hline Q7 & 0.034 & 0.878 & 0.088 & 0.119 & -0.047 & 0.046 & 0.798 \\
\hline Q8 & 0.103 & 0.860 & 0.091 & 0.203 & -0.012 & 0.014 & 0.799 \\
\hline Q9 & 0.009 & 0.842 & 0.079 & 0.059 & 0.025 & -0.014 & 0.720 \\
\hline Q10 & 0.080 & 0.680 & 0.028 & -0.193 & 0.230 & 0.076 & 0.565 \\
\hline Q11 & 0.043 & 0.638 & -0.055 & -0.118 & 0.202 & 0.292 & 0.552 \\
\hline Q12 & -0.009 & 0.073 & 0.837 & 0.102 & 0.194 & 0.013 & 0.754 \\
\hline Q13 & 0.056 & -0.006 & 0.724 & 0.243 & 0.004 & 0.087 & 0.595 \\
\hline Q14 & 0.148 & 0.131 & 0.701 & -0.143 & 0.090 & 0.304 & 0.652 \\
\hline Q15 & 0.218 & 0.090 & 0.645 & 0.160 & 0.201 & -0.243 & 0.597 \\
\hline Q16 & 0.344 & 0.086 & 0.624 & -0.127 & 0.131 & 0.166 & 0.575 \\
\hline Q17 & 0.177 & -0.061 & 0.491 & 0.168 & 0.242 & 0.241 & 0.421 \\
\hline Q18 & 0.340 & 0.047 & -0.010 & 0.722 & 0.155 & 0.095 & 0.672 \\
\hline Q19 & 0.341 & 0.072 & 0.244 & 0.639 & 0.030 & 0.348 & 0.710 \\
\hline Q20 & 0.507 & 0.021 & 0.128 & 0.573 & 0.032 & 0.195 & 0.641 \\
\hline Q21 & 0.396 & 0.012 & 0.139 & 0.560 & 0.311 & 0.179 & 0.618 \\
\hline Q22 & 0.396 & 0.008 & 0.104 & 0.469 & 0.423 & 0.062 & 0.570 \\
\hline Q23 & 0.189 & 0.167 & 0.213 & -0.028 & 0.780 & 0.056 & 0.721 \\
\hline Q24 & 0.171 & 0.113 & 0.280 & 0.203 & 0.721 & 0.249 & 0.743 \\
\hline Q25 & 0.297 & 0.077 & 0.174 & 0.245 & 0.719 & 0.026 & 0.702 \\
\hline Q26 & 0.147 & 0.145 & 0.030 & 0.050 & 0.229 & 0.747 & 0.656 \\
\hline Q27 & 0.308 & 0.124 & 0.224 & 0.283 & 0.052 & 0.720 & 0.762 \\
\hline Q28 & 0.279 & 0.078 & 0.188 & 0.306 & -0.020 & 0.650 & 0.636 \\
\hline
\end{tabular}

anemia subscales ofBrief Fatigue Inventory (BFI) [13], the Functional Assessment of Cancer Therapy instrument (FACT-F), Bidimensional Fatigue Scale (BFS) [15, 16], European Organization for Research and Treatment of Cancer QOL Questionnaire (EORTC QLQ-C30) and Multidimensional Fatigue Symptom Inventory-Short Form (MFSI-SF) [17]: fatigue subscale [18]. However, trusty and appropriate instrument for measuring fatigue and CRF cognition is essential for successful clinical care of Taiwanese patients with cancers who experience this troubling symptom. Because fatigue is subjective, clinicians must rely on patient self-reports to assess its presence and severity, which can then be supported by additional sources of information such as physical examination, laboratory data, and family members' descriptions. In addition, the results of a questionnaire differ in different societies [19-21]. Lack of equivalent measures has been one of the difficulties encountered by researchers intending to perform cross-cultural research [20-22]. Most researchers could not ensure the equivalence of a translated measure's back translation. Although basic methods may ensure translational 
Table 3: Exploratory factor analysis of 6 dimensions of the Taiwan cancer-related fatigue questionnaire

\begin{tabular}{|c|c|c|c|c|c|c|}
\hline \multicolumn{7}{|c|}{ CRF summary with the maximum variation method } \\
\hline $\begin{array}{c}\text { New items } \\
\text { (re-edited ordering) }\end{array}$ & Unfocused life & $\begin{array}{l}\text { Attribution } \\
\text { cognition }\end{array}$ & $\begin{array}{c}\text { Help } \\
\text { expectation }\end{array}$ & $\begin{array}{c}\text { Treatment } \\
\text { helplessness }\end{array}$ & $\begin{array}{l}\text { Physician -patient } \\
\text { communication }\end{array}$ & $\begin{array}{c}\text { Life } \\
\text { power }\end{array}$ \\
\hline Eigen values & 4.764 & 3.271 & 3.27 & 2.68 & 2.433 & 2.336 \\
\hline Reserved items & 6 & 5 & 6 & 5 & 3 & 3 \\
\hline Explained variance & 17.013 & 11.682 & 11.666 & 9.572 & 8.689 & 8.344 \\
\hline Cumulative amount of explained variance & 66.966 & & & & & \\
\hline Subscale Cronbach's $\alpha$ & 0.910 & 0.852 & 0.809 & 0.847 & 0.814 & 0.794 \\
\hline Total table Cronbach's $\alpha$ & 0.933 & & & & & \\
\hline Adequacy of sampling (KMO) & 0.868 & & & & & \\
\hline Bartlett's spherical test $\chi^{2}$ value & $\begin{array}{c}\chi^{2}(378, N=148)= \\
2390.11, p<0.001\end{array}$ & & & & & \\
\hline
\end{tabular}

Table 4: Four exploratory factor analyses of the Taiwan cancer-related fatigue questionnaire

\begin{tabular}{ccccc}
\hline EFA times & KMO $>\mathbf{0 . 4}$ & Aspect numbers & $\begin{array}{c}\text { Numbers of deleted } \\
\text { items }\end{array}$ & Deleted items \\
\hline 1 & 0.868 & 7 & 3 & 7 \\
& & 8 & & 6 \\
2 & 0.867 & 7 & 2 & 8 \\
3 & 0.863 & 7 & 2 & 16 \\
4 & 0.868 & 6 & 0 & 15 \\
\hline
\end{tabular}

equivalence, the translated edition and original measures perform in the equivalent of psychometrically manners might be different [19-21]. Because of different cultures, languages, and social classes [23], developing a Taiwanese CRF cognition questionnaire is crucial to the investigation of CRF and fatigue-related cognition in the future. According to our review of the literature, the TCRFCQ-V1.0 is the first instrument for estimating CRF and fatigue-related cognition in Taiwanese patients with cancers. We suggest that in the future, all patients with cancers should be screened for CRF cognition at the initial visit, during cancer treatments, and every 3 months after treatments. Screening patients with cancers at different therapeutic intervals can be crucial to understanding the optimal treatment time for CRF in these patients.

Fatigue is a common problem in patients with cancers and occurs in patients receiving aggressive cancer therapy as well as in cancer patients who have completed all cancer treatment. Progressive cancer growing, cytotoxic CT, targeted therapy, biological response modifiers, RT or CCRT, anemia, pain, poor nutrition emotional distress, and sleep disturbance were contributed to CRF. QOL of both patients and their families were affected deeply by CRF, whatever psychosocial, physical and even occupational or economic fields [4-6, 24]. However, in contemporary oncological treatment, the main issue of supportive care are the treatment and screening of CRF. Screening for CRF in cancer patients are recommended based on American Society of Clinical Oncology and NCCN guidelines $[1,25]$. If any of the factors associated with fatigue is identified, these should be addressed as an initial approach to fatigue. Optimizing the management of CRFassociated symptoms such as pain, nausea, and dyspnea can help in alleviating fatigue and further improving the QOL of both patients and their families. The QOL of patients with cancer without CRF can be improved and optimal cancer treatment can be provided if they report being out of symptoms of fatigue.

Our tool seems reliable, feasible and validity in view of the preliminary data of psychometric measurements. First, we believe that the questionnaire satisfactorily covers the sentiences of CRF expressed by cancer patients under study because no cancer patient gained the lowest or highest probable entire score and dimension score. Besides it indicates that the measure, at least theoretically, can express poor or good changes with proper tolerance in all dimensions $(<15 \%$ cancer patients have extreme score in the total score or any of the dimentions) [26]. Second, the completion rate is good $(97 \%)$, which is calculated as the rate of cancer patients not missing responses in any retained question, was quite satisfactory. A group of 43 items was to evaluate CRF in cancer patients and each 
item was assessed in terms of importance and frequency. And there were only some items selected for cancer patients. The quantity of information lost during the performance of questionnaire would be low. The content of this type of questionnaire in Taiwanese cancer patients would be acceptable according to our results. Regarding the cause and effect (reflexivity) of the qualitative data, the TCRFCQ-V1.0 includes 6 dimensions of CRF. The naming of the dimensions was based on the original questions in similar psychological concepts. We named these dimensions on the basis of our own clinical and academic experience; thus, we believe that our study is creative and original.

As listed in Table 1, The satisfaction of Cronbach alpha coefficients were achieved. Over the suggested minimum of 0.70 of the Cronbach alpha values for all the dimensions and the total score were reached for use at the questionnaire. [27]. This type of questionnaire used in individual level should be over 0.90 in the total score [26]. This is especially crucial because developing of a Taiwanese questionnaire which could assess CRF in cancer patient was our goal. After cancer patients who answered 43 items twice, the reported values were gained within the context of the item reduction study. Cancer patients completing the final version of the questionnaire and required to respond to each of the items only once would improve the quality of our questionnaire. The reliability of the TCRFCQ-V1.0 was satisfactory in our study.

$\mathrm{CRF}$ is a distressing psychological condition experienced by patients with cancer, and CRF cannot be easily measured. A psychometric approach is suitable for evaluating the emotions of people. EFA, which was used in the study, is a statistical method that can reveal the basic structure of fatigue. In this study, we reviewed the literature in MEDLINE ${ }^{\circledR}$, PubMed $^{\circledR}$, National Cancer Institute, and Chinese academic databases to design and analyze the TCRFCQ-V1.0 as well as to connect the psychological knowledge base with the clinical understanding of cancers. The feasible of factor analysis were presented well according to the significant results of Bartlett's test (chisquared, 2390.11; $p<0.001$; Table 3). The KMO statistic of 0.868 also implied the adequacy of sampling. All KMO statistics of EPA (Table 4) were significant. Compared with the defined groups, the known groups showed coherent between the expected range after the validity analysis. The TCRFCQ-V1.0 includes 6 dimensions of CRF, namely unfocused life, attribution cognition, help expectation, treatment helplessness, physician-patient communication, and life power. These 6 factors accounted for $67 \%$ of the total variance (Table 3 ). The inclusion of the $6 \mathrm{CRF}$ dimensions in the TCRFCQ-V1.0 is a strength of this study. The 6 factors are different from those included in previous CRF questionnaires such as FACT-F, BFI [13], BFS [15, 16], MFSI-SF [17], and EORTC QLQ-C30: fatigue subscale [18]. The depth and breadth of the TCRFCQ-V1.0 are more extensive.
There are some limitations in the current study. Although the completion rate of the TCRFCQ-V1.0 was high ( $97 \%$ of the patients completed all questions) and missing ratewas low $(0.2 \%-1.1 \%$ for each question), a small amount of misleading information may be present in our study. Furthermore, the patient sample studied may not be the deputy of the general population of Taiwanese cancer patients, because the cancer patients recruited were from a single institute in Taiwan. Moreover, $87 \%$ of the patients had a high education level (at least high school). Thus, the extrapolation of the TCRFCQ-V1.0 to patients having a low education level might be insufficient.

\section{Conclusions}

The TCRFCQ-V1.0 has satisfactory internal consistency and reliability and is a convenient-to-use clinical measure of fatigue cognition in Taiwanese cancer patients. In the future, the prevalence of CRF and fatigue-related cognition among Taiwanese patients with cancer will be investigated. The refinement and use of the TCRFCQ-V1.0 can help in supporting CRF cognition research and clinical management, in addition to improving communication between patients and clinicians.

\section{MATERIALS AND METHODS}

\section{Item generation}

We reviewed the literature in $M E D L I N E^{\circledR}, \mathrm{PubMed}^{\circledR}$, National Cancer Institute, and Chinese academic databases to design and analyze the TCRFCQ-V1.0 as well as to connect the psychological knowledge base with the clinical understanding of cancers. The responses of the TCRFCQ-V1.0 were recorded using a 5-point response scale (strongly disagree, disagree, ordinary, agree, or very much agree). More than $90 \%$ of patients selected the lowest or highest category, and the initial 43 items in the TCRFCQ-V1.0 were thus assessed for floor and ceiling effects, respectively. Items with these effects were removed from further analysis (3 items). The final items in the TCRFCQ-V1.0 were selected on the basis of a combination of statistical evidence and clinical relevance. The total score was the summation of the retained items. Higher scores indicated higher levels of CRF cognition.

\section{Patients}

We enrolled patients with cancer who were aged $>18$ years, had received a pathological diagnosis of cancer, and had undergone cancer treatments such as surgery, chemotherapy (CT), radiotherapy (RT), and concurrent chemoradiotherapy (CCRT) at a single institute in Taiwan. Our protocols were reviewed and approved by the Institutional Review Board of Taipei City Hospital (TCHIRB-1021103-E). The enrolled patients were 
asked to complete the 43-item, investigator-designed TCRFCQ-V1.0 (See Supplementary Table 1 for the version translated into English). The survey was conducted by trained study nurses in the outpatient department of a single institute. After informed consent was obtained from patients, they were enrolled in the study.

\section{Questionnaire}

The TCRFCQ-V1.0 (the initial 43-item version) was verbally delivered by trained interviewers and was completed through personal interviews for all patients $(100 \%)$. The interviewers received standardized training, and interviews were monitored for consistency across study sites. The patients were provided a hard copy of response categories, and the interviewer recorded their responses. Although the mentioned tools have not been specifically tested, they have been extensively validated within the Taiwan population. Additionally, these tools were used during the early stages of TCRFCQ-V1.0 development, and preliminary evidence determines them to be suitable with respect to content and language.

\section{Statistical analysis}

Statistical analyses were conducted using SPSS software (Version 20; IBM Corporation, Armonk, NY). We used descriptive statistics to summarize the demographic and clinical characteristics of the patients and the prevalence of CRF cognition. Furthermore, we used Bartlett's test of sphericity and the KaiserMeyer-Olkin (KMO) measure of sampling adequacy to examine the appropriateness of the sample size for conducting exploratory factor analysis (EFA). Factors with eigenvalues of $>1$ were identified using principal component analysis, and scree plots were used to determine the point at which the decrease in eigenvalues became negligible. A 6-factor model was selected because it was the most clinically meaningful model. Furthermore, we conducted EFA, forcing a 6-factor solution with varimax rotation. The conventional primary factor loading cutoff value of $\geq 0.55$ was used to identify items for retention, which were then attributed to the factor with the highest loading. Items within identified factors were assessed for their internal consistency by using Cronbach's alpha reliability coefficients. Items for which the patients reported $\geq 15 \%$ moderate to high levels of cognition but had loadings that were lower than the cutoff value were retained due to clinical significance. The convergent validity of the total cognition score was tested using the Spearman rank-order correlation and the Pearson correlation coefficient. Discriminant validity was assessed by comparing median CRF scores by using the MannWhitney or Kruskal-Wallis test.

\section{CONFLICTS OF INTEREST}

None.

\section{REFERENCES}

1. Bower JE, Bak K, Berger A, Breitbart W, Escalante CP, Ganz PA, Schnipper HH, Lacchetti C, Ligibel JA, Lyman GH, Ogaily MS, Pirl WF, Jacobsen PB. Screening, assessment, and management of fatigue in adult survivors of cancer: an American Society of Clinical oncology clinical practice guideline adaptation. Journal of clinical oncology. 2014; 32:1840-1850.

2. Bower JE, Ganz PA, Desmond KA, Rowland JH, Meyerowitz BE, Belin TR. Fatigue in breast cancer survivors: occurrence, correlates, and impact on quality of life. Journal of clinical oncology. 2000; 18:743-753.

3. Servaes P, Verhagen S, Bleijenberg G. Determinants of chronic fatigue in disease-free breast cancer patients: a crosssectional study. Annals of oncology. 2002; 13:589-598.

4. Bloom JR, Fobair P, Gritz E, Wellisch D, Spiegel D, Varghese A, Hoppe R. Psychosocial outcomes of cancer: a comparative analysis of Hodgkin's disease and testicular cancer. Journal of clinical oncology. 1993; 11:979-988.

5. Dagnelie PC, Pijls-Johannesma MC, Lambin P, Beijer S, De Ruysscher D, Kempen GI. Impact of fatigue on overall quality of life in lung and breast cancer patients selected for high-dose radiotherapy. Annals of oncology. 2007; 18:940-944.

6. Gupta D, Lis CG, Grutsch JF. The relationship between cancer-related fatigue and patient satisfaction with quality of life in cancer. Journal of pain and symptom management. 2007; 34:40-47.

7. Stasi R, Abriani L, Beccaglia P, Terzoli E, Amadori S. Cancer-related fatigue: evolving concepts in evaluation and treatment. Cancer. 2003; 98:1786-1801.

8. Winningham ML. Strategies for managing cancer-related fatigue syndrome: a rehabilitation approach. Cancer. 2001; 92:988-997.

9. Cella D, Peterman A, Passik S, Jacobsen P, Breitbart W. Progress toward guidelines for the management of fatigue. Oncology (Williston Park). 1998; 12:369-377.

10. Cella D, Davis K, Breitbart W, Curt G. Cancer-related fatigue: prevalence of proposed diagnostic criteria in a United States sample of cancer survivors. Journal of clinical oncology. 2001; 19:3385-3391.

11. Howell D, Keller-Olaman S, Oliver TK, Hack TF, Broadfield L, Biggs K, Chung J, Gravelle D, Green E, Hamel M, Harth T, Johnston P, McLeod D, et al. A panCanadian practice guideline and algorithm: screening, assessment, and supportive care of adults with cancerrelated fatigue. Curr Oncol. 2013; 20:e233-246.

12. Mitchell SA, Beck SL, Hood LE, Moore K, Tanner ER. Putting evidence into practice: evidence-based interventions 
for fatigue during and following cancer and its treatment. Clinical journal of oncology nursing. 2007; 11:99-113.

13. Mendoza TR, Wang XS, Cleeland CS, Morrissey M, Johnson BA, Wendt JK, Huber SL. The rapid assessment of fatigue severity in cancer patients: use of the Brief Fatigue Inventory. Cancer. 1999; 85:1186-1196.

14. Wu HS, McSweeney M. Assessing fatigue in persons with cancer: an instrument development and testing study. Cancer. 2004; 101:1685-1695.

15. Alexander S, Minton O, Stone PC. Evaluation of screening instruments for cancer-related fatigue syndrome in breast cancer survivors. Journal of clinical oncology. 2009; 27:1197-1201.

16. Chalder T, Berelowitz G, Pawlikowska T, Watts L, Wessely S, Wright D, Wallace EP. Development of a fatigue scale. Journal of psychosomatic research. 1993; 37:147-153.

17. Stein KD, Jacobsen PB, Blanchard CM, Thors C. Further validation of the multidimensional fatigue symptom inventory-short form. Journal of pain and symptom management. 2004; 27:14-23.

18. Minton O, Stone P. A systematic review of the scales used for the measurement of cancer-related fatigue (CRF). Annals of oncology. 2009; 20:17-25.

19. Meisenberg G, Williams A. Are acquiescent and extreme response styles related to low intelligence and education? Personality and Individual Differences. 2008; 44 :1539-1550.

20. Fischer R. Standardization to Account for Cross-Cultural Response Bias: A Classification of Score Adjustment Procedures and Review of Research in JCCP. Journal of Cross-Cultural Psychology. 2004; 35:263-282.
21. Nederhof AJ. Methods of coping with social desirability bias: A review. European Journal of Social Psychology. 1985; 15:263-280.

22. Ferrer M, Alonso J, Prieto L, Plaza V, Monso E, Marrades R, Aguar MC, Khalaf A, Anto JM. Validity and reliability of the St George's Respiratory Questionnaire after adaptation to a different language and culture: the Spanish example. The European respiratory journal. 1996; 9:1160-1166.

23. Beaton DE, Bombardier C, Guillemin F, Ferraz MB. Guidelines for the process of cross-cultural adaptation of self-report measures. Spine. 2000; 25:3186-3191.

24. Spelten ER, Verbeek JH, Uitterhoeve AL, Ansink AC, van der Lelie J, de Reijke TM, Kammeijer M, de Haes JC, Sprangers MA. Cancer, fatigue and the return of patients to work-a prospective cohort study. Eur J Cancer. 2003; 39:1562-1567.

25. Dy SM, Lorenz KA, Naeim A, Sanati H, Walling A, Asch SM. Evidence-based recommendations for cancer fatigue, anorexia, depression, and dyspnea. Journal of clinical oncology. 2008; 26:3886-3895.

26. McHorney CA, Tarlov AR. Individual-patient monitoring in clinical practice: are available health status surveys adequate? Quality of life research. 1995; 4:293-307.

27. Passik SD, Kirsh KL, Donaghy K, Holtsclaw E, Theobald D, Cella D, Breitbart W. Patient-related barriers to fatigue communication: initial validation of the fatigue management barriers questionnaire. Journal of pain and symptom management. 2002; 24:481-493. 